\title{
Componentes Sensoriais e Atencionais do Tempo de Reação: Efeitos do Tamanho, Excentricidade e Previsibilidade de Estímulos Visuais
}

\author{
Luiz Renato Rodrigues Carreiro ${ }^{1}$ \\ Universidade Presbiteriana Mackenzie \\ Hamilton Haddad \\ Universidade de São Paulo \\ Marcus Vinicius Chrysostomo Baldo \\ Universidade de São Paulo
}

\begin{abstract}
RESUMO - A percepção visual depende do arcabouço sensorial e do processamento atencional. Este trabalho estudou o efeito, sobre o tempo de reação manual (TR), do tamanho, excentricidade e previsibilidade de estímulos visuais. No experimento 1 $(\mathrm{n}=8)$, um alvo foi apresentado aleatoriamente em uma de quatro excentricidades diferentes, possuindo três possíveis tamanhos O experimento $2(\mathrm{n}=12)$ apresentava configuração similar, porém uma pista indicava o quadrante de maior probabilidade $(70 \%)$ de apresentação do alvo. Os resultados mostraram um aumento do TR em função da excentricidade do alvo, além de uma diminuição do TR com o aumento do tamanho do alvo e indicação correta da pista. Uma análise das interações sugere uma superposição de mecanismos atencionais e puramente sensoriais compartilhando um estágio comum do processamento visual.
\end{abstract}

Palavras-chave: Atenção visual, Excentricidade, Tamanho, Tempo de Reação, Percepção

\section{Sensory and Attentional Components of Reaction Time: Effects of the Size, Eccentricity and Predictability of Visual Stimuli}

\begin{abstract}
Visual perception depends on the sensory framework and on attentional processing. This study examined the effects of the size, eccentricity and predictability of a visual stimulus on manual reaction time (RT). In experiment $1(\mathrm{n}=8)$, a target was presented randomly in one of four different eccentricities, with three possible sizes. Experiment $2(n=12) \mathrm{had}$ a similar configuration but a cue indicated the higher predictability (70\%) of target presentation quadrant. The results showed an increase in RT as a function of target eccentricity, and a decrease in RT with increasing target size or accuracy of the cue. An analysis of interaction effects suggests an overlay of attentional and purely sensory mechanisms sharing common stages of visual processing.
\end{abstract}

Keywords: Visual attention, Eccentricity, Size, Reaction Time, Perception

O processamento ativo das informações sensoriais permite a interação eficiente dos organismos com o meio e consequentemente sua atuação mais adaptativa. De acordo com Helmholtz (1867) a percepção visual pode ser compreendida como um processo de inferência da mais provável situação ambiental, dado um padrão de estimulação. Esse processamento depende tanto do arcabouço sensorial quando de fatores atencionais. $\mathrm{O}$ arcabouço sensorial é necessário para que os estímulos ambientais sejam eficientemente captados e processados pelo sistema nervoso. Já a atenção, possibilita a seleção de determinados estímulos para que estes possam ser melhor processados, impedindo processamentos desnecessários pelo sistema nervoso (Steinman \& Steinman, 1998). Tal processo de seleção ou filtragem é denominado de atenção seletiva (Desimone \& Duncan, 1995). Knudsen (2007) descreve também a correlação da atenção com outros processos cognitivos (como memória operacional, o controle descendente (top-down) da sensibilidade, o processamento

1 Endereço para correspondência: Universidade Presbiteriana Mackenzie, Centro de Ciências Biológicas e da Saúde, Programa de Pós-Graduação em Distúrbios do Desenvolvimento, Rua da Consolação, 896. Prédio 38-Térreo. Consolação, SP. CEP 01302-000. E-mail: luizrenato.carreiro@mackenzie.br automático ascendente (bottom-up) de filtragem de estímulos) que, de modo integrado, possibilitam a seleção de informações do ambiente.

Desde o final do século XIX (James, 1890), a psicologia experimental tem estudado os fenômenos atencionais, demonstrando a possibilidade de orientação da atenção visual sem movimento dos olhos, ou seja, sem que se direcione a fóvea para sua posição (para uma revisão, ver Carrasco, 2006). Desta forma, estabelece-se uma dissociação entre a fixação do olhar (para onde explicitamente se olha) e a do foco atencional (para onde se presta atenção). Posner (1978, 1980) estudou respostas manuais a estímulos luminosos com o objetivo de saber se a emissão dessas respostas seria mais rápida se os participantes soubessem antecipadamente o local em que estes estímulos apareceriam.

Nesses estudos, as imagens retinianas dos estímulos (sejam localizados à direita ou à esquerda do ponto de fixação) formavam-se sempre a uma mesma distância da fóvea. Desse modo, qualquer diferença observada entre os tempos de reação (TR) nas condições de orientação correta (quando o alvo aparece na posição indicada), neutra (quando não há indicação prevendo a posição do alvo) e incorreta (quando o alvo aparece em outra posição que não a indicada) não 
dependeria de fatores retinianos ou motores (uma vez que a resposta solicitada era a mesma: pressionar uma tecla), mas deveria ser causada por mecanismos centrais envolvidos com a expectativa da ocorrência do estímulo em certa posição do espaço. Tal efeito de validade da pista também foi encontrado em tarefas de busca visual (Galera, Cavallet, Von Grunau, \& Panagopoulos, 2006). Trabalhos recentes também têm buscado estudar, por meio de procedimentos semelhantes aos de Posner (1978; 1980), sinais de desatenção e hiperatividade (Araújo \& Carreiro, 2009) ou efeitos de treino atencional avaliando, por exemplo, jogadores do voleibol e não esportistas em tarefas de orientação voluntária e automática da atenção (Carreiro, Ferreira, \& Machado-Pinheiro, 2009) demonstrando que tais tipos de experimentos podem identificar habilidades comprometidas ou facilitadas de direcionamento da atenção.

O desempenho de um participante em tarefas TR não depende apenas de processamentos elaborados da informação sensorial, o que inclui a alocação ou desvios da atenção, mas também das características definidas pelo próprio processamento sensorial desta informação. Por exemplo, a fóvea, porção central da retina que ocupa de $1,5^{\circ}$ a $2^{\circ}$ de ângulo visual, difere em vários aspectos das porções retinianas mais periféricas: há nela maior número de fotorreceptores; o tamanho dos campos receptivos é menor e verifica-se menor relação fotorreceptores/célula ganglionar, gerando menor convergência (Rodieck, 1998; Masland, 2001; Field \& Chichilnisky, 2007).

Esses fatores intrínsecos ao sistema visual podem por si mesmos, sem que haja influência de fatores atencionais, levar a diferenças nos TR. Isso pode acontecer, por exemplo, quando o alvo visual ocorre em diferentes excentricidades ou quando o tempo de duração da apresentação do alvo é ampliado ou reduzido, refletindo processos relacionados à organização anátomo-funcional do sistema visual, como descrito anteriormente. Tal relação tem sido descrita em muitos trabalhos que investigam a influência, sobre o TR, de fatores tais como a intensidade (Cattel, 1886; Froeberg, 1907; Piéron, 1920, 1952; Rains, 1963; Pins \& Bonnet, 1997), o tamanho (Osaka, 1976; Bonnet, Gurlekian, \& Harris, 1992) e a localização de estímulos no campo visual (Osaka, 1982; Mancebo-Azor, Saez-Moreno, Dominguez-Hidalgo, Luna-Del Castillo, \& Rodriguez-Ferrer, 2009; Carrasco, Evert, Chang, \& Katz, 1995, Carrasco, Giordano, \& McElree, 2006), além da participação de mecanismos atencionais (Posner, 1978; Tassinari \& Berlucchi, 1995; Palmer, 1999; Liu, Stevens, \& Carrasco, 2007).

No entanto, não existe, ainda, uma clara distinção entre o papel da alocação da atenção e o papel de mecanismos puramente sensoriais envolvidos na latência de uma resposta motora à estimulação visual. Um estímulo apresentado na periferia do campo visual associa-se, em geral, a maiores latências da resposta motora. Isso pode ser resultado tanto de mecanismos sensoriais básicos, como os relacionados ao processamento retiniano diferenciado para regiões distintas do campo visual quanto do tempo consumido para desvios atencionais no campo visual. Carreiro, Haddad e Baldo (2011) estudaram o efeito de diferentes intensidades de estímulos visuais sobre o TR a alvos em diferentes condições de previsibilidade e ocu- laridade (binocular e monocular). Foi observado, por tais autores, que o TR diminui a medida que a intensidade do alvo aumenta, quando se passa de uma condição monocular para binocular ou quando a previsibilidade do alvo aumenta, entretanto não foram observadas interações dos fatores sensorias e atencionais.

Osaka (1976) apresentou estímulos de diferentes tamanhos e em diferentes excentricidades para estudar a influência destes fatores sobre o TR. Os resultados demonstram que o TR diminui em função do aumento do tamanho do alvo e que a magnitude desta queda foi menor na periferia do que na fóvea. Estudando os determinantes do TR a estímulos luminosos que variavam em área e luminância, Bonnet et al (1992) verificaram que o TR declina quando o tamanho do estímulo luminoso aumenta. $\mathrm{O}$ efeito encontrado por Bonnet é explicado como sendo um efeito de somação de probabilidade; ao aumentar a área e a intensidade do estímulo, aumenta também a atividade neuronal no sistema visual, o que pode levar à diminuição do TR.

Carrasco, Evert, Chang e Katz (1995), Carrasco, McLean, Katz e Frieder (1998), Carrasco e Yeshurun (1998), Carrasco (2006), Carrasco, Giordano e McElree (2006) estudaram a influência da excentricidade no desempenho de participantes em tarefas de busca visual. Em tais trabalhos observou-se que, com o aumento da excentricidade há também aumento dos TR e do número de erros. Resultado semelhante também foi observado na acurácia da detecção de alvos visuais periféricos: ela deteriora com o aumento da excentricidade da apresentação do alvo (Chan $\&$ Courtney 1998). Além disto, verificou-se também que a detectabilidade de uma grade de estimulação pode ser similar quando apresentada em diferentes excentricidades se ela for corrigida, na visão periférica, como proporção inversa do fator de magnificação cortical da distribuição dos fotorreceptores e células ganglionares (Rovamo \& Virsu, 1979; Virsu, Näsänen e Osmovita 1987).

Mancebo-Azor et al (2009) estudaram os efeitos do contraste, excentricidade e posição de estímulos visuais apresentados na retina central. Tais autores verificaram que o contraste e a excentricidade são determinantes na detecção de estímulos visuais, já que há um aumento dos TR em função da excentricidade e uma diminuição dos TR em função do aumento do contraste do alvo.

Existem, portanto, diversos trabalhos que investigaram os efeitos de características psicofísicas de alvos visuais e de sua previsibilidade sobre o TR. Entretanto, ainda são necessários estudos que busquem elucidar as contribuições e, sobretudo, as interações de fatores sensoriais e atencionais sobre a resposta manual. Assim, o presente trabalho tem como objetivo analisar os efeitos do tamanho, excentricidade e previsibilidade de um estímulo visual sobre o TR manual. Para tanto, foram planejados dois experimentos, o primeiro teve como objetivo descrever a influência do tamanho e da excentricidade de um alvo visual sobre o TR e o segundo, compreender os efeitos da orientação prévia da atenção sobre o TR a alvos visuais de diferentes tamanhos apresentados em diferentes excentricidades. 


\section{Método}

\section{Participantes}

Participaram do experimento 1 oito voluntários (três homens e cinco mulheres), que se submeteram a três sessões experimentais. No experimento 2 tomaram parte doze voluntários (três homens e nove mulheres), sendo que cada um participou de sete sessões experimentais (duas sessões para cada uma das três excentricidades, além de uma primeira sessão utilizada como treino). Todos os participantes eram alunos de graduação ou pós-graduação, possuíam acuidade visual normal ou corrigida e estavam na faixa etária de 18 a 30 anos (experimento 1:22 $\pm 3,8$ d.p. e experimento $2: 22,5 \pm$ 3,2 d.p.). Com relação ao número de estímulos apresentados aos participantes eles foram 240 no experimento 1 e 384 no experimento 2, divididos, tanto em um quanto em outro, em 4 blocos que levavam, em média, de 25 a 30 minutos. Antes do início da série de testes, cada participantes respondeu a um questionário de avaliação geral (para identificação de uso de medicamentos, problemas neurológicos, uso de lentes corretivas), e foi informados sobre o tipo de experimento, de sua finalidade acadêmica e respectivos procedimentos, assinando o termo de consentimento livre e esclarecido.

\section{Instrumentos}

Foram realizados testes de tempo de reação utilizando rotinas computacionais elaboradas por meio do software "Micro Experimental Laboratory - MEL" (MEL Professional 2.01- Psychology Software Tools, Inc.). No experimento 1 cada teste se iniciava com a apresentação de um ponto de fixação (PF) no centro da tela. Após um intervalo variável de 800 a $1800 \mathrm{~ms}$ era apresentado um quadrado em uma de quatro excentricidades possíveis $\left(1,2^{\circ}, 4,5^{\circ}, 9,0^{\circ}\right.$ e $\left.13,5^{\circ}\right)$, em cada um dos quatro quadrantes visuais, e com três tamanhos diferentes possíveis: $0,2^{\circ}, 0,5^{\circ}$ e $0,9^{\circ}$ (Figura 1A). A cada apresentação, os participantes não sabiam com qual tamanho ou em que excentricidade e quadrante o estímulo apareceria, já que não havia nenhum tipo de indicação que informasse sobre a ocorrência específica de qualquer uma dessas características. No experimento 2 cada apresentação iniciava-se com o surgimento do PF, juntamente com o qual era apresentada uma linha apontando para um dos quatro quadrantes da tela. Esta linha indicava que naquele quadrante havia uma maior probabilidade $(70 \%)$ de apresentação do alvo e uma probabilidade menor (30\%) do estímulo aparecer em qualquer um dos outros três quadrantes. Em outra condição, o PF era sobreposto a um asterisco, indicando que a probabilidade era a mesma $(25 \%)$ do estímulo aparecer em qualquer quadrante. Esse experimento foi baseado nos procedimentos utilizados por Posner (1980). Após um intervalo variável de 800 a $1800 \mathrm{~ms}$, era apresentado o alvo (Figura 1C). Como no experimento 1, havia três tamanhos possíveis para o alvo: $0,2^{\circ}, 0,5^{\circ}$ e $0,9^{\circ}$ de lado, selecionados aleatoriamente a cada apresentação. Além disto, a cada sessão de testes esses estímulos eram apresentados em uma dentre 3 possíveis excentricidades $\left(4,5^{\circ}, 9,0^{\circ}\right.$ ou $\left.13,5^{\circ}\right)$. As excentricidades eram fixas por sessões de teste para que cada participante pudesse orientar a atenção para a exata posição de aparecimento do alvo.

Tanto no experimento 1 quanto no experimento 2, todos os participantes foram instruídos a manter o olhar no PF e a responder o mais rapidamente possível ao aparecimento do alvo, pressionando uma chave óptica com o dedo indicador da mão dominante, independente do tamanho ou posição do estímulo apresentado. No caso específico do experimento 2 os participantes deveriam, também, sem mover os olhos, orientar a atenção para a posição indicada pela linha. Após o participante emitir a resposta, era apresentado na tela o seu TR em milissegundos, a indicação de antecipação (quando a resposta era emitida antes do aparecimento do alvo) ou de resposta lenta (quando se passava $1000 \mathrm{~ms}$ do aparecimento do alvo sem a emissão da resposta).

\section{Procedimentos}

Em uma sessão de testes, cada participante sentava-se em frente a um monitor de tela plana de 15 polegadas acoplado a um computador, que gerava os estímulos e registrava as respostas. As sessões experimentais foram realizadas em uma sala com atenuação sonora e luz ambiente diminuída e indireta. A cabeça do participante ficava posicionada em um apoiador de fronte e mento para que a distância entre seus olhos e a tela permanecesse constante a $50 \mathrm{~cm}$. Os procedimentos metodológicos aqui descritos (tanto para o experimento 1 quanto para o experimento 2) foram submetidos e aprovados pelo Comitê de Ética em Pesquisa Envolvendo Seres Humanos.

\section{Resultados}

\section{Experimento 1}

Após o término das sessões experimentais, foi calculada a mediana dos TR em cada condição para cada um dos oito participantes. Os dados foram, então, submetidos a uma análise de variância (ANOVA) com medidas repetidas para os fatores (1) excentricidade (4 níveis: $1,2^{\circ}$, $4,5^{\circ}, 9,0^{\circ}$ e $13,5^{\circ}$ ) e (2) tamanho (3 níveis: $0,2^{\circ}, 0,5^{\circ}$ e $0,9^{\circ}$ de lado). Como resultados, diferenças estatisticamente significativas foram verificadas para os fatores excentricidade $\left[F(3,21)=15,7 ; p<0,0001, \eta^{2}\right.$ parcial $\left.=0,692\right] \mathrm{e}$ tamanho $\left[F(2,14)=76,2 ; p<0,0001, \eta^{2}\right.$ parcial $\left.=0,916\right]$, não havendo interações significativas desses fatores. Os resultados indicaram que o TR diminui sistematicamente com o aumento do tamanho do alvo. Esses resultados podem ser confirmados pelas análises post-hoc (utilizando o método de Tukey), verificando-se significativo um efeito do fator tamanho (com $\mathrm{p}<0,01)$ para todos os três tamanhos em todas as excentricidades. Além disso, verificou-se também aumento do TR em função da excentricidade da apresentação do alvo, principalmente quando se comparam as excentricidades de $1,2^{\circ}$ e $4,5^{\circ} \mathrm{com}$ a de $13,5^{\circ}$ cuja análise post-hoc (utilizando o método de Tukey) demonstrou 

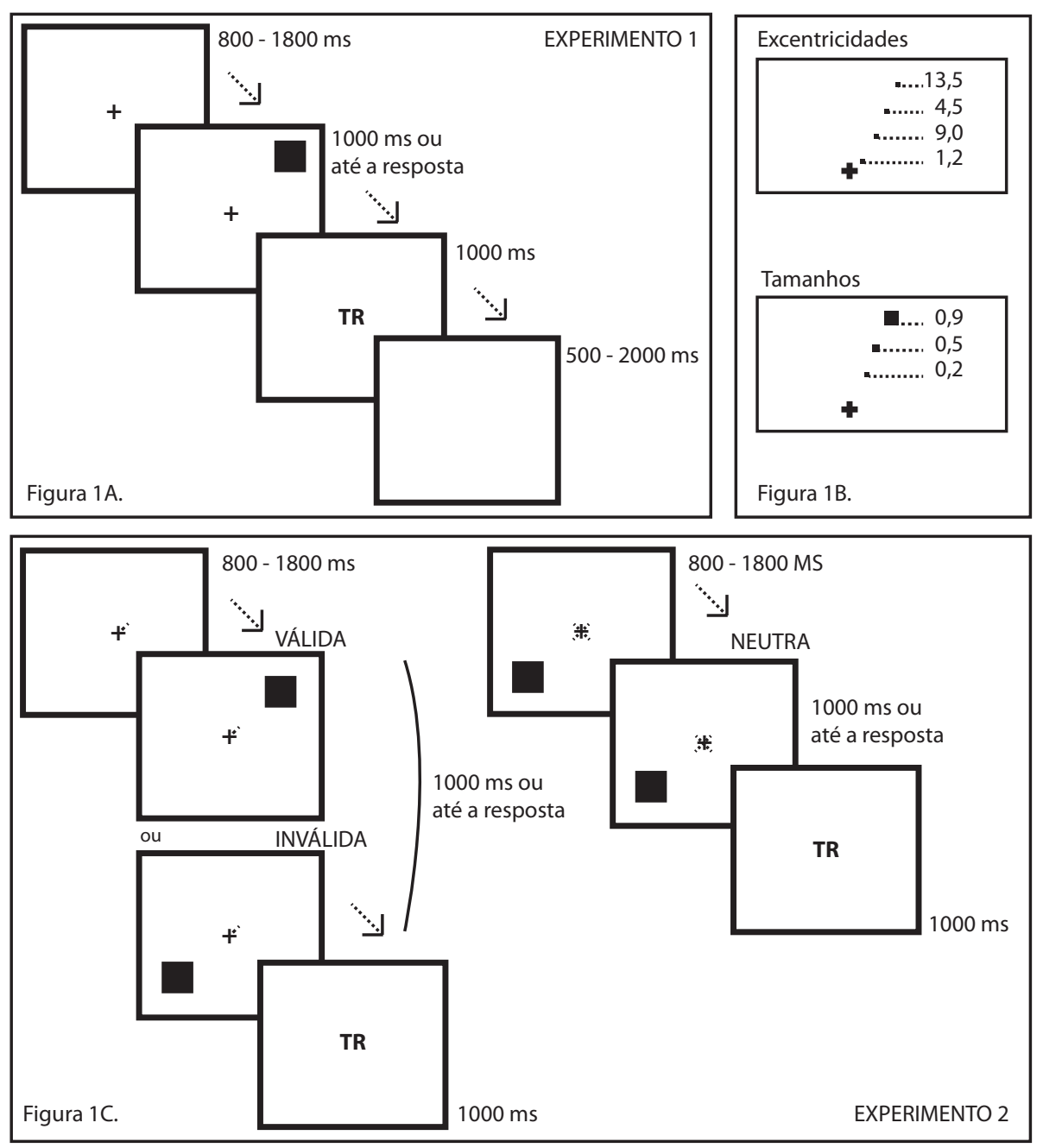

Figura 1. Sequência temporal da apresentação dos estímulos nos experimentos 1 (Figura 1A) e 2 (Figura 1C) no qual são representadas também as situações válida, neutra e inválida. A figura 1B descreve as excentricidades e tamanhos (ambos expressos em graus) utilizados nos experimentos.

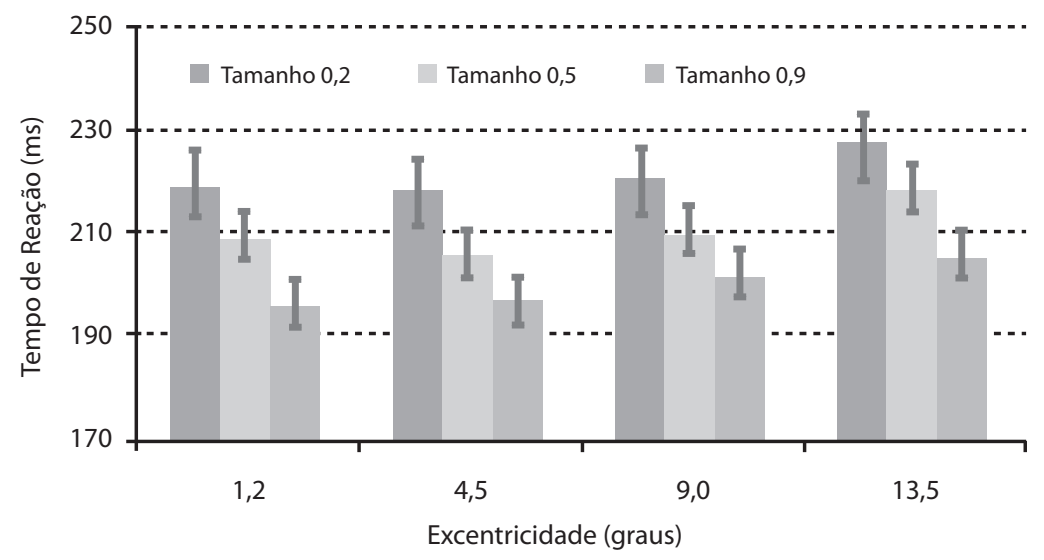

Figura 2. Tempo de Reação (Média \pm EPM) para as diferentes excentricidades e diferentes tamanhos de alvos no experimento 1 . 
diferenças com $\mathrm{p}<0,05$ para todos os tamanhos (Figura 2). Alem disso, foi realizado também o teste de contraste na ANOVA para o experimento 1 , que demonstrou uma tendência significativa do tipo linear $(p=0,001)$ e uma tendência significativa do tipo quadrática $(\mathrm{p}=0,003)$ para o fator excentricidade, além de uma tendência significativa do tipo linear $(\mathrm{p}<0,001)$ para o fator excentricidade, descrevendo assim que o TR aumenta tanto com o aumento da excentricidade quanto com a diminuição do tamanho.

\section{Experimento 2}

Após o término das sessões experimentais, foi calculada a mediana dos TR em cada condição para cada um dos doze participantes. Os dados foram, então, submetidos a uma análise de variância (ANOVA) com medidas repetidas com os fatores (1) excentricidade (3 níveis: $4,5^{\circ}, 9,0^{\circ}$ e $13,5^{\circ}$ ), (2) tamanho (3 níveis: $0,2^{\circ}, 0,5^{\circ}$ e $0,9^{\circ}$ de lado) e (2) pista (3 níveis: válida, neutra e inválida). Análises post-hoc utilizando o método de Tukey foram utilizadas para comparações individuais.

Essa análise demonstrou um efeito significativo do fator tamanho $\left[\mathrm{F}(2,22)=385,9 ; \mathrm{p}<0,0001, \eta^{2}\right.$ parcial $\left.=0,972\right]$ sobre o TR, que diminui à medida que o tamanho do alvo aumenta (para todos os tamanhos com valores de $\mathrm{p}<0,001$ de acordo com a análise post-hoc), de modo semelhante ao experimento 1. Houve também efeito significativo do fator pista $\left[F(2,22)=46,4 ; p<0,0001, \eta^{2}\right.$ parcial $\left.=0,808\right]$ sobre o TR, sendo este menor na condição válida, maior na condição inválida e intermediário na condição neutra, com valores de $\mathrm{p}<0,001$ de acordo com a análise post-hoc. Os resultados para esse experimento podem ser vistos na Figura 3. O efeito do fator excentricidade ocorreu com um nível de significância marginal $\left[F(2,22)=3,1 ; p=0,0661, \eta^{2}\right.$ parcial $=0,219]$, sendo os TR da excentricidade $9,0^{\circ}$ diferentes dos da excentricidade $13,5^{\circ}$ com $p=0,053$, de acordo com a análise post-hoc. Além disso, foi realizado o teste de contraste na ANOVA que demonstrou uma tendência significativa do tipo quadrática $(\mathrm{p}=0,023)$ para o fator excentricidade, e uma tendência significativa do tipo linear $(\mathrm{p}<0,001)$ para o fator excentricidade, descrevendo assim que o TR aumenta tanto com o aumento da excentricidade quanto com a diminuição do tamanho. No caso do fator pista, observa-se também tendência significativa do tipo linear $(p<0,001)$, descrevendo uma diminuição do TR em função da informação fornecida por ela. Observou-se, também, uma interação significativa dos fatores tamanho e pista $\left[F(4,44)=7,5 ; p<0,0001, \eta^{2}\right.$ parcial $=0,406]$, principalmente pelo efeito da pista inválida, cujo TR aumenta com a diminuição do tamanho de forma mais evidente do que com os outros tipos de pistas, como será discutido a seguir.

Ao serem utilizadas pistas válidas, inválidas e neutras, é possível considerar os TR obtidos com a pista neutra como uma linha de base, definindo, a partir dela, custos e benefícios atencionais, como descrito pelos trabalhos de Posner $(1978,1980)$. Custos são definidos como a diferença entre os TR obtidos nas condições neutra e inválida $\left(\right.$ Custo $\left.=\mathrm{TR}_{\text {neutra }}-\mathrm{TR}_{\text {inválida }}\right)$. Já os benefícios são definidos pela diferença entre os TR nas condições neutra e válida $\left(\right.$ Benefício $\left.=\mathrm{TR}_{\text {neutra }}-\mathrm{TR}_{\text {válida }}\right)$. Por essa razão, em geral, os benefícios resultam em valores positivos, enquanto os custos resultam negativos.

$\mathrm{Na}$ comparação custo/benefício realizada a partir dos resultados do experimento 2 , observa-se que o benefício não se modificou significativamente nem com o aumento do tamanho nem com o aumento da excentricidade do alvo; já o custo apresenta uma tendência a diminuir com o aumento do tamanho e a aumentar com o aumento da excentricidade (Tabela 1). Uma análise de variância multivariada (MANOVA) das variáveis dependentes "benefício" e "custo" revelou um efeito significativo do fator Tamanho $(\mathrm{p}=0,0072)$ mas não do fator excentricidade $(\mathrm{p}=0,3582)$. Seguindo-se uma análise post-hoc (utilizando o método de Tukey), verificou-se que o efeito do fator tamanho decorre de sua influência sobre os custos $(p=0,0163$, para a comparação entre os tamanhos extremos), mas não sobre os benefícios $(\mathrm{p}>0,10)$.

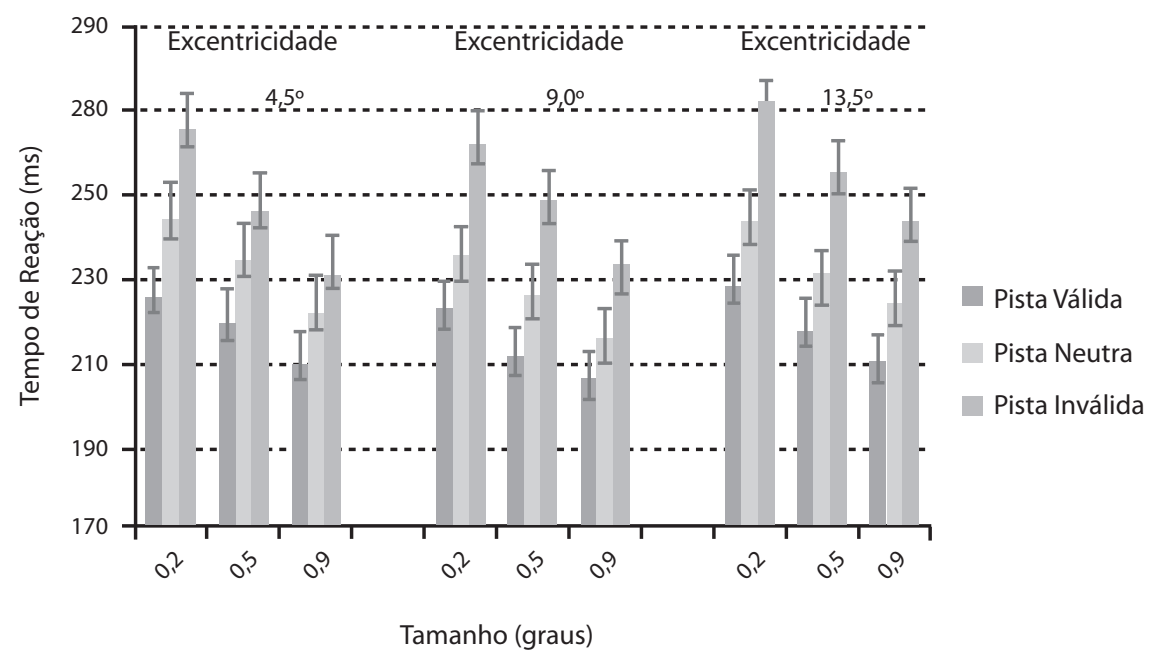

Figuras 3. Tempo de reação (Média \pm EPM) para os diferentes tamanhos e excentricidades do alvo para as condições de pista válida, neutra e inválida. 
Tabela 1. Custos e benefícios atencionais em função do tamanho e excentricidade do estímulo no experimento 2 .

\begin{tabular}{cccc}
\hline $\begin{array}{c}\text { TAMANHO } \\
\text { (Graus) }\end{array}$ & $\begin{array}{c}\text { EXCENTRICIDADE } \\
\text { (Graus) }\end{array}$ & $\begin{array}{c}\text { BENEFÍCIO } \\
(\mathrm{ms})\end{array}$ & $\begin{array}{c}\text { CUSTO } \\
(\mathrm{ms})\end{array}$ \\
\hline \multirow{2}{*}{0,2} & 4,5 & 19,3 & $-22,2$ \\
& 9,0 & 13,2 & $-27,4$ \\
& 13,5 & 16,2 & $-27,1$ \\
& & & \\
0,5 & 4,5 & 15,2 & $-12,0$ \\
& 9,0 & 14,9 & $-23,9$ \\
& 13,5 & 14,1 & $-22,5$ \\
& & & $-18,4$ \\
0,9 & 4,5 & 12,9 & $-19,1$ \\
\hline
\end{tabular}

\section{Discussão}

Osaka $(1976,1982)$ descreveu aumentos do TR em função da excentricidade, correlacionado esse fato à relativa diminuição da densidade de cones na periferia da retina em relação à fóvea. $\mathrm{O}$ efeito da excentricidade sobre o TR tem sido também estudado em tarefas de busca visual. Carrasco et al (1995) encontraram um pronunciado efeito da excentricidade descrevendo que à medida que ela aumentava o TR e os erros também aumentavam gradativamente. Desse modo, os resultados observados no experimento 1 estão de acordo com os dados encontrados na literatura.

Dados semelhantes à diminuição do TR como função do aumento do tamanho do alvo também são encontrados na literatura (Bonnet et al., 1992; Osaka, 1976). Bonnet et al (1992) utilizam, inclusive, diferentes níveis de luminância para o alvo, observando um decréscimo do TR em função da área para todos os níveis de luminância estudados. De acordo com a teoria de magnificação cortical (Virsu et al., 1987; Rovamo \& Virsu, 1979), a visibilidade de estímulos é similar sobre o campo visual desde que as representações corticais dos estímulos, calculadas por meio do fator de magnificação, sejam similares. Assim, um estímulo maior na periferia pode levar a uma resposta semelhante a um estímulo menor mais próximo a fóvea.

Além da influência dos fatores sensoriais no TR, dados da literatura têm demonstrado a influência de fatores atencionais na sua modulação. Posner et al (1980) sugerem que a alocação da atenção intensifica o processamento precoce da informação visual, produzindo um efeito facilitador que reduz a latência de detecção. Hughes (1984) parte da premissa que um efeito da atenção sobre mecanismos precoces de processamento deveria conduzir a uma interação significativa de fatores atencionais e sensoriais, como por exemplo, a intensidade do estímulo. Desse modo, ainda persiste o debate questionando se os mecanismos atencionais interferem na seleção precoce ou tardia de características do estímulo a serem processadas com maior resolução (Hughes, 1984; Hawkins et al., 1988, 1990; Luck et al., 1994).
Para compreender melhor a participação de fatores atencionais e sensorias sobre o TR foi executado o experimento 2. Nesse experimento, as diferenças encontradas entre as condições válida, neutra e inválida reproduzem os resultados encontrados por Posner $(1978,1980)$ e por Rizzolatti, Riggio, Dascola e Umiltà (1987), mostrando que a latência da resposta a estímulos visuais é afetada pela previsibilidade espacial do estímulo. Observou-se, também, uma interação significativa dos fatores tamanho e pista, demonstrando que apesar da diminuição do tamanho levar a um aumento do TR em todos os níveis do fator pista, no caso da pista inválida observa-se um maior aumento do TR com a diminuição do tamanho, em comparação às outras situações atencionais. Com relação à interação dos fatores pista e excentricidade, observa-se também que a situação inválida leva a um maior aumento no TR com o aumento da excentricidade, em comparação às outras situações atencionais. Essas interações estão estreitamente vinculadas às relações entre as magnitudes do custo e benefício atencionais e os fatores tamanho e excentricidade. Ou seja, uma maior dependência do custo em relação, por exemplo, ao tamanho do estímulo, associa-se ao fato de um maior efeito desse fator sobre a situação de pista inválida, conduzindo a uma interação significativa. Analisando-se, portanto, as relações custo/benefício, observa-se uma tendência do benefício não se modificar (ou se modificar menos) com a excentricidade ou com o tamanho, enquanto o custo apresentou uma nítida dependência do tamanho do estímulo, estatisticamente significativa.

As interações significativas dos fatores pista e tamanho e dos fatores pista e excentricidade observadas no experimento 2 indicam, segundo a lógica dos fatores aditivos (Sternberg, 1969), que mecanismos atencionais (manipulados pelas pistas simbólicas) e sensoriais (manipulados pelo tamanho e excentricidade do estímulo) devem exercer suas influências sobre pelo menos um estágio comum de processamento visual. Com esse tipo de raciocínio, os resultados desse trabalho podem ser acomodados partindo-se de um modelo conceitual que associa o TR a três tipos principais de latências, decorrentes de estágios sucessivos de processamento: a primeira, aqui denominada de latência sensorial $\left(\mathrm{L}_{\mathrm{S}}\right)$, estaria condicionada a latências originadas da condução da informação pelas vias sensoriais; outra, denominada de latência atencional $\left(\mathrm{L}_{\mathrm{A}}\right)$, estaria vinculada ao grau de ativação necessária para o adequado processamento do estímulo, e apresentada pelos respectivos circuitos neurais envolvidos; e um terceiro componente, uma latência motora $\left(\mathrm{L}_{\mathrm{M}}\right)$, seria gerada pelo processamento motor e execução do movimento, aqui suposta constante. $\mathrm{O}$ equacionamento do TR como resultado de uma sequência de processos ou estágios sucessivos, onde um se inicia após o término do anterior, remonta ao século XIX, com o trabalho de Donders (1868/1969).

Assim, $\mathrm{TR}=\mathrm{L}_{\mathrm{S}}+\mathrm{L}_{\mathrm{A}}+\mathrm{L}_{\mathrm{M}}$. Em nosso modelo, $\mathrm{L}_{\mathrm{S}}$ seria dependente das características físicas do estímulo (tamanho, excentricidade, luminância), podendo ser expresso pela função $\mathrm{L}_{\mathrm{S}}=\mathrm{f}_{\mathrm{S}}$ (estímulo). Por outro lado, o grau de ativação de um dado circuito, aqui supostamente determinante de $\mathrm{L}_{\mathrm{A}}$, seria dependente tanto das características do estímulo quanto da orientação voluntária ou automática da atenção de um indivíduo. Ou seja, poderia ser expresso pela função $\mathrm{L}_{\mathrm{A}}=\mathrm{f}_{\mathrm{A}}($ estímulo, orientação). 
No caso de uma pista válida, a orientação voluntária da atenção ativaria previamente os circuitos destinados ao processamento do estímulo, reduzindo a latência atencional $\left(\mathrm{L}_{\mathrm{A}}\right)$ a um valor mínimo aproximadamente constante nas várias circunstâncias experimentais. Nesse caso, características do estímulo, tais como tamanho, luminância, excentricidade, reteriam fundamentalmente seus efeitos sobre a latência sensorial $\left(\mathrm{L}_{\mathrm{S}}\right)$, já que a facilitação atencional dos circuitos envolvidos naquele processamento estaria maximizada, minimizando $\mathrm{L}_{\mathrm{A}}$. No caso de uma pista inválida, o estímulo encontraria os respectivos circuitos neurais responsáveis pelo processamento não apenas sem qualquer facilitação prévia, mas com recursos atencionais eventualmente drenados para os circuitos invalidamente facilitados. Nesse caso, a facilitação desses circuitos iria se beneficiar de qualidades favoráveis do estímulo (como maiores tamanhos ou intensidades, ou ainda menores excentricidades). Essas características psicofísicas do estímulo exerceriam, portanto, não só um efeito sobre a latência sensorial $\left(\mathrm{L}_{\mathrm{S}}\right)$, como antes, mas agora também sobre a latência atencional $\left(\mathrm{L}_{\mathrm{A}}\right)$, não mais levada a um mínimo irredutível pela orientação prévia da atenção, mas situada em um nível maior provocado pela situação inválida, e capaz de se beneficiar dessas características psicofísicas. Esse modelo, portanto, explica razoavelmente o maior efeito das características do estímulo sobre a situação de pista inválida e, da mesma forma, o maior efeito observado para o custo atencional, em comparação ao benefício. Lembramos que os efeitos sobre as relações custo/benefício estão diretamente relacionadas às interações dos fatores tamanho e pista, e dos fatores pista e excentricidade.

Os resultados obtidos nos experimentos desse trabalho estão de acordo com Downing e Pinker (1985) que mostraram também que os custos aumentam com o aumento da excentricidade. Posner (1978) obteve resultados distintos, tendo observado que tanto os custos quanto os benefícios de uma orientação atencional voluntária foram os mesmos, sem expressar qualquer efeito da excentricidade dos estímulos. Downing e Pinker (1985) sugerem que estas discrepâncias possam estar relacionadas ao número de possíveis posições para o alvo (Posner utilizou duas posições, enquanto Downing e Pinker utilizaram 10 posições). Em nosso caso, utilizamos 4 coordenadas angulares (quadrantes) e 3 radiais (excentricidades), totalizando 12 possíveis posições.

Os resultados observados aqui também se alinham aos obtidos por Hawkins et al $(1988,1990)$ e Luck et al (1994), que também obtiveram interações significativas de fatores atencionais e fotométricos. Esses autores concordam com a proposta de Posner, em que a atenção visual teria um locus de ação sensorial. No entanto, não concebem os diferentes padrões espaço-temporais da distribuição da atenção como decorrentes de operações de desengajamento, movimento e engajamento do foco atencional. Segundo eles, o efeito de pistas visuais na detecção de um estímulo, caracterizado pela sua luminância, é atribuído a diferenças na ativação de estruturas sensoriais facilitadas por pistas válidas ou não facilitadas por pistas inválidas.

Os resultados desse trabalho permitem conceber a atenção visual como um processo que continua, às vezes se superpondo, ao processamento sensorial. Nesse sentido, muitos aspectos que são descritos como sensoriais ou atencionais podem depender de mecanismos interagentes e associados a um mesmo contínuo de processamento, sem que uma linha divisória nítida possa ser traçada com exatidão (Summerfield \& Egner, 2009). Enquanto os efeitos da luminância do estímulo têm início nos fotorreceptores, os efeitos atencionais provavelmente surgem em estágios mais tardios de processamento, como observado também por evidências no trabalho de Carreiro, Haddad e Baldo de 2011. No entanto, talvez seja ainda uma questão prematura a determinação de um estágio comum, seja precoce ou tardio, em que essa interação ocorre.

\section{Referências}

Araujo, R. R., \& Carreiro, L. R. R. (2009). Orientação voluntária e automática da atenção e indicadores de desatenção e hiperatividade em adultos. Avaliação Psicológica, 8, 325-336.

Bonnet, C., Gurlekian, J., \& Harris, P. (1992). Reaction time and visual area: Searching for the determinants. Bulletin of the Psychonomic Society, 30, 396-398.

Carrasco, M., Evert, D., Chang, I., \& Katz, S. (1995). The eccentricity effect: Target eccentricity affects performance on conjunction searches. Perception \& Psychophysics, 57, 1241-1261.

Carrasco, M., McLean, T. L., Katz, S. M., \& Frieder, K. S. (1998). Feature asymmetries in visual search: Effects of display duration, target eccentricity, orientation \& spatial frequency. Vision Research, 38, 347-374.

Carrasco, M., \& Yeshurun, Y. (1998). The contribution of covert attention to the set-size and eccentricity effects in visual search. Journal of Experimental Psychology: Human Perception \& Performance, 24, 673-692.

Carrasco, M. (2006). Covert attention increases contrast sensitivity: Psychophysical, neurophysiological, and neuroimaging studies. In S. Martinez-Conde, S. L. Macknik, L. M. Martinez, J. M. Alonso, \& P. U. Tse (Eds), Visual Perception. Part I. Fundamentals of Vision: Low and Mid- Level Processes in Perception, Progress in Brain Research, Elsevier, 154, 33-70.

Carrasco, M., Giordano, A. M., \& McElree, B. (2006). Attention speeds processing across eccentricity: Feature and conjunction searches. Vision Research, 46, 2028-2040.

Carreiro, L. R. R., Ferreira, I. R., \& Machado-Pinheiro, W. (2009). Comparação de desempenho de jogadores de voleibol e não esportistas em tarefas de orientação automática e voluntária da atenção visual: Um estudo exploratório. Psicologia: Teoria e Prática, 11, 38-49.

Carreiro, L. R. R., Haddad, H., \& Baldo, M. V. (2011). Effects of intensity and positional predictability of a visual stimulus on simple reaction time. Neuroscience Letters, 10, 345-349.

Cattell, J. M. (1886). The influence of the intensity of the stimulus on the length of the reaction time. Brain, 9, 512-515.

Chan, H. S., \& Courtney, A. J. (1998). Stimulus size scaling and foveal load as determinants of the peripheral target detection. Ergonomics, 41, 1433-1452.

Desimone, R., \& Duncan, J. (1995). Neural mechanisms of selective visual attention. Annual Review of Neuroscience, 18, 193-222.

Donders, F. C. (1969). On the speed of mental process, 1868. Traduzido em: W.G. Koster (Ed.), Attention and Performance II, Acta Psychologica, 30, 412-431. 
Downing, C. J., \& Pinker, S. (1985). The spatial structure of visual attention. In M. I. Posner \& O. S. M. Martin (Eds.), Attention and performance XI (pp. 171-187). Hillsdale. NJ: Erlbaum.

Field, G. D., \& Chichilnisky, E. J. (2007). Information processing in the primate retina: Circuitry and coding. Annual Review Neuroscience, 30, 1-30.

Froeberg, S. (1907). The relation between the magnitude of stimulus and the time of reaction. Archives of Psychology, New York, 16, 1-38.

Galera, C., Cavallet, M., Von Grunau, M., \& Panagopoulos, A. (2006). Características atentivas reveladas por dicas múltiplas locais e globais. Psicologia: Teoria e Pesquisa, 22, 327-334.

Hawkins, H. L., Shafto, M. G., \& Richardson, K. (1988). Effects of Target Luminance and Cue Validity on the Latency of Visual Detection Latency. Perception \& Psychophysics, 44, 484-492.

Hawkins, H. L., Hillyard, S. A., Luck, S. J., Downing, C. J., Mouloua, M., \& Woodward, D. P. (1990). Visual Attention Modulates Signal Detectability. Journal of Experimental Psychology: Human Perception and Performance, 16, 802-811.

Helmholtz, H. von (1867/1925). Treatise in Physiological Optics (From 3rd German Edition, Trans.) (3rd Ed., Vol. III). New York: Dover Publications.

Hughes, H. C. (1984). Effects of flash luminance and positional expectance on visual response latency. Perception \& Psychophysics, 36, 177-184.

James, W. (1890). Principles of Psychology. New York: Holt.

Knudsen, E. I. (2007). Fundamental components of attention. Annual Review of Neuroscience, 30, 57-78.

Liu, T., Stevens, S. T., \& Carrasco, M. (2007). Comparing the time course and efficacy of spatial and feature-based attention. Vision Research, 47, 108-113.

Luck, S. J., Hillyard, S. A., Mouloua, M., Woldorff, M. G., Clark, V. P., \& Hawkins, H. J. (1994). Effects of spatial cuing on luminance detectability: Psychophysical and eletrophysiological evidence for early selection. Journal of Experimental Psychology: Human Perception and Performance, 20, 887-904.

Mancebo-Azor, R., Saez-Moreno, J. A., Dominguez-Hidalgo, I., Luna-Del Castillo, J. D., \& Rodriguez-Ferrer, J. M. (2009). Efectos del contraste, excentricidad y posición en la detección de estímulos visuales en humanos. Revista de Neurología, 48, 129-133.

Masland, R. H. (2001). The fundamental plan of the retina. Nature Neuroscience, 4, 877-886.

Osaka, N. (1976). Reaction time as a function of peripheral retinal locus around fovea: effect of stimulus size. Perception \& Motor Skills, 43, 603-606.

Osaka, N. (1982). Exponent of the latency and brightness power functions in the fovea and periphery of the visual field. Journal of General Psychology, 106, 195-203.
Palmer, S. E. (1999). Vision science. MIT Press, Cambridge.

Piéron, H. (1920). Nouvelles research sur l'analyse du temps de latence sensorielle en fonction des intensités excitatrices. L'Anne Psychologique, 22, 58-142.

Piéron, H. (1952). The sensations. New Haven, Yale University Press.

Pins, D., \& Bonnet, C. (1997). Reaction times reveal the contribution of different receptor components in luminance perception. Psychonomic Bulletin and Review, 4, 359-366.

Posner, M. I. (1978). Chronometric exploration of mind. Lawrence Erlbaum Associates, Hillsdale, New Jersey.

Posner, M. I. (1980). Orienting of attention. Quaterly Journal of Experimental Psychology, 32, 3-25.

Rains, J. D. (1963). Signal luminance and position effects in human reaction time. Vision Research, 3, 239-251.

Rizzolatti, G., Riggio, L., Dascola, I., \& Umiltà, C. (1987). Reorienting attention across the horizontal and vertical meridians: evidence in favor of a premotor theory of attention. Neuropsychologia, 25, 31-40.

Rodieck, R.W. (1998). The first steps in seeing. Sinauer Associates, Inc, Massachusetts.

Rovamo, J., \& Virsu, V. (1979). An estimation and application of the human cortical magnification factor. Experimental Brain Research, 37, 495-510.

Steinman, S. B., \& Steinman, B. A. (1998). Vision and attention. I: Current models of visual attention. Optometry and Vision Science, 75, 146-155.

Sterneberg, S. (1969). The discovery of processing stages: Extensions of Donder's method. In W.G. Koster (Ed.), Attention and performance II (pp. 276-315). Amsterdam: North-Holland.

Summerfield, C., \& Egner, T. (2009). Expectation (and attention) in visual cognition. Trends in Cognitive Sciences, 13, 403-409.

Tassinari, G., \& Berlucchi, G. (1995). Covert orienting to noninformative cues: reaction time studies. Behavioral Brain Research, 71, 101-112.

Virsu, V., Näsänen, R., \& Osmovita, K. (1987). Cortical magnification and peripheral vision. Journal of the Optical Society of America, 4, 1568-1577.

Recebido em 05.04.2010

Primeira decisão editorial em 14.09.2011

Aceito em 05.12.2011 